\title{
The Trend Towards the Right (Proximal) Shift of Colorectal Cancer: Is Not Observed in Sudanese Patients
}

\author{
Hyder Osman Mirghani ${ }^{1}$, Ali Adam Elhadi ${ }^{2}$, Ibrahim Abdullah Albalawi ${ }^{3}$ \\ ${ }^{1}$ Department of Internal Medicine, Faculty of Medicine, University of Tabuk, Tabuk, Kingdom of Saudi Arabia \\ ${ }^{2}$ Department of Internal Medicine, Medical College, Omdurman Islamic University, Omdurman, Sudan \\ ${ }^{3}$ Department of Surgery, Faculty of Medicine, University of Tabuk, Tabuk, Kingdom of Saudi Arabia
}

\section{Email address:}

drbalawi@yahoo.com (I. A. Albalawi)

\section{To cite this article:}

Hyder Osman Mirghani, Ali Adam Elhadi, Ibrahim Abdullah Albalawi. The Trend Towards the Right (Proximal) Shift of Colorectal Cancer: Is Not Observed in Sudanese Patients. American Journal of Clinical and Experimental Medicine. Vol. 4, No. 5, 2016, pp. 156-159. doi: 10.11648/j.ajcem.20160405.18

Received: September 6, 2016; Accepted: September 21, 2016; Published: October 18, 2016

\begin{abstract}
Background: There is a tendency of colorectal cancer towards a right shift, and increasing incidence among the young age groups. In this study, we aimed to assess the pattern of colorectal cancer among Sudanese patients attending an endoscopy unit in Omdurman Teaching Hospital. Subjects and Methods: This descriptive cross- sectional study conducted among thirty patients referred with the provisional diagnosis of colorectal cancer during the period from August 2015 to June 2016. Participants signed a written informed consent then interviewed to collect demographic data, symptoms related to carcinoma of the colon, the patients were then examined for evidence of anemia, intestinal obstruction, and ascites, colonoscopy with biopsy for histopathology was done. The ethical committee of the Omdurman Teaching Hospital approved the research, and the Statistical Package for Social Sciences (SPSS) was used for data analysis. Results; Out of thirty patients with the diagnosis of colorectal cancer, their ages ranged from 18-76 years with a mean of 51.1. The commonest presentations were rectal bleeding, change in the bowel habits, and constipation in $90 \%, 80 \%$, and $60 \%$ respectively. Fourteen $(46.7 \%$ were $\leq 50$ years. The family history of colorectal cancer was evident in $16.7 \%$, the recto-sigmoid area was the commonest site $(83.3 \%)$, with $100 \%$ adenocarcinoma. Conclusion: Colorectal cancer tend to affect the younger age groups, the majority were recto-sigmoid (the proximal shift was not observed). The adenocarcinoma was the commonest histopathology.
\end{abstract}

Keywords: Proximal Shift, Colorectal Cancer, Sudan

\section{Introduction}

Cancer is a leading cause of morbidity and mortality worldwide, an estimated number of 12.7 million occurred in the year 2008 with 715000 new cases. The number is expected to double by the year 2030[1]. Colorectal cancer is amongst the most common cancers worldwide with an upsurge in the incidence in the resource-limited countries [2]

Colorectal cancer is ranked as the fourth most common cancers among men and the second among women [3]. In Sudan the most common cancers among both sexes were breast, non-Hodgkin lymphoma, leukemia, esophagus, and colorectal [1]. Registries result from 2009-2010 in Khartoum; Sudan reported that the colorectal cancer was ranked the fifth among all primary tumors in the Capital of Sudan [4].
There is a trend towards the declining or stabilization in the incidence of colorectal cancer in the developed world attributed to the implementation of an organized screening program with opposite upward trends for those under the age of 50 years [5].

There is an increasing concern about the growing aging populations and the change in the lifestyle (the adoption of diet with high fat and red meat and lack of physical activity) may translate in a higher incidence of colorectal cancer [6].

Cancer continues to receive low priority in Sudan and Africa as a whole, despite the growing burden worldwide, which could be attributed to limited resources especially for health, political instability, and other pressing heath issues 
like infectious diseases, [malaria, tuberculosis, and Acquired Immunodeficiency Syndrome) [7], furthermore there is a lack of awareness among the policy makers as well as the public about the current and future burden of cancer ${ }^{1}$. The matter is further complicated by the trend toward the proximal shift of colorectal cancer in the developed world with the need for more expensive invasive investigations (colonoscopy, and barium studies) as the optimal techniques for pro [per detection of these cancers instead of digital examination and proctosigmoidoscopy [8].

Sudan is a vast country with ethnic and social diversity, health services when present is located in the major cities, the pattern of the carcinoma of the colon may differ from others in the developed world. Thus we conducted this research to assess the pattern of the carcinoma of the colorectal area in Omdurman Teaching Hospital in Sudan.

\section{Subjects \& Methods}

This cross-sectional perspective Hospital based study conducted at the endoscopy unit in Omdurman Teaching Hospital, Sudan during the period from August 2015 to June 2016. Thirty patients referred for lower GI endoscopy with the provisional diagnosis of carcinoma of the colon were recruited. Participants were invited to sign a written informed consent form, then interviewed by the assigned physician to collect: demographic data, symptoms of anemia, rectal bleeding, chronic constipation, change in the bowel habit for 6 weeks or more, loose stool or increase frequency of stool for 6 weeks, participant were also interviewed for the fulfillment of hereditary polyposis and hereditary non- polyposis, family history of carcinoma of the colon in the first degree relatives, and the type of treatment received, the patient were then examined to confirm pallor, presence of ascites, and signs of intestinal obstruction. A fiberopticcolonoscopy Olympus UK was used to view the recto-sigmoid, ascending, transverse, the descending colon, and cecum. Biopsies were taken for histopathology The standard technique of the instrument sterilization, patient positioning, and follow-up was followed. The patient was assured that the data collected would be treated confidentially and for the purpose of research only.

The ethical committee of the Omdurman Teaching Hospital approved the research, and the Statistical Package for Social Sciences (SPSS) was used for data analysis, the data were presented as percentages or mean \pm sd unless otherwise specified. AP-value of $<0.05$ was considered significant.

\section{Results}

Out of thirty-five patients with carcinoma of the colon $50 \%$ were women, their ages ranged from 18-76 years with a mean of \pm sd, $40 \%$ were from Omdurman (Ombadda), $16.7 \%$ from Aljazeera, $13.3 \%$ from Kurdufan, $10 \%$ were coming from Darfur and Alshimaliya, while White Nile, Blue Nile, and Gadharif regions reported 3.3\% for each. Near half of the patients were homemakers $46.7 \%$, followed by farmers

\section{$22.3 \%$ Table (1).}

Rectal bleeding was the commonest presentation of the patients $(90 \%)$, followed by change in the bowel habit in $(80 \%), 60 \%$ of the present sample presented with constipation and symptoms of anemia, 30 showed increase in the frequency of stool, abdominal masses were detected in $23.3 \%$, intestinal obstruction in $20 \%$, and ascites in $6.7 \%$ of patients. In the current data hereditary, polyposis coli was diagnosed in $10 \%$ of patients, while $6.7 \%$ of patients reported a family history of carcinoma of the colon in their firstdegree relatives Table (2). Synchronous tumors, metachronous tumors, and hereditary non-polyposis were not detected in the present study (data not shown).

It is interesting to note that the majority $(83.3 \%)$ of carcinoma of the colon were involving the recto-sigmoid area, followed by the descending colon $(13.3 \%)$, the cecum and ascending colon in a minority of patients $(3.3 \%)$, while the tumor was not detected in the transverse colon. Table (3).

Table (4) depicted the type of treatment received in which; The majority of patients $(83.3 \%)$ received surgery \& radiotherapy, surgery alone was reported in $10 \% \%$ of patients, chemotherapy alone in $6.7 \%$, while only $3.3 \%$ received radiation only.

Table 1. Characteristics of the study group.

\begin{tabular}{ll}
\hline Character & $\%$ \\
\hline Age years & $18-76$ \\
Range & 51.03 \\
Mean \pm sd & \\
Sex & 50 \\
Males & 50 \\
Females & \\
Residence & 40 \\
Omdurman & 16.7 \\
Gazera & 13.3 \\
Kurdfan & 10 \\
Darfur & 10 \\
Shimaliya & 3.3 \\
White Nile & 3.3 \\
Blue Nile & 3.3 \\
Gadharif & \\
Occupation & 46.7 \\
Housewife & 22.3 \\
farmer & 13.3 \\
Laborer & 10 \\
Student & 6.7 \\
Clerk & \\
\hline
\end{tabular}

Table 2. The clinical presentation of patients with Ca colon.

\begin{tabular}{ll}
\hline Character & \%No \\
\hline Rectal bleeding & $27(90)$ \\
Change in bowel habit & $24(80)$ \\
Chronic constipation & $18(60)$ \\
Symptoms of anemia & $18(60)$ \\
Increase in stool frequency & $9(30)$ \\
Abdominal mass & $7(23.3)$ \\
Intestinal obstruction & $6(20)$ \\
Ascites & $2(6.7)$ \\
Hereditary polyposis coli & $3(10)$ \\
Family history of Ca colon & $2(6.7)$ \\
Adenocarcinoma & $30(100 \%)$ \\
Age $\leq 50$ years & $14(46.7 \%)$ \\
\hline
\end{tabular}


Table 3. The site of Ca colon among the study group

\begin{tabular}{ll}
\hline Site & No\% \\
\hline Caecum and ascending colon & $1(3.3)$ \\
Transverse colon & $0(0)$ \\
Descending colon & $4(13.3)$ \\
Recto-sigmoid area & $25(83.3)$ \\
\hline
\end{tabular}

Table 4. The type of treatment among patients with Ca colon.

\begin{tabular}{ll}
\hline The kind of treatment & No \% \\
\hline Surgery & $3(10)$ \\
Radiotherapy & $1(3.3)$ \\
Chemotherapy & $2(6.7)$ \\
Surgery+ Radiotherapy & $25(83.3)$ \\
\hline
\end{tabular}

\section{Discussion}

A trend towards the proximal migration of colonic cancer, and the increasing incidence among the younger age group had been reported in different areas worldwide $[5,6]$.

In the present study the majority $(83.3 \%)$ of the colonic cancer was located in the rectosigmoid area, with an only minority in the cecum and ascending colon, similar studies reported that there is an increasing incidence of all colonic cancers instead of right shift or proximally [9]. Previous literature reported a migration of colonic cancer proximally in contradiction to the current data, increasing the age of the population and the change of the lifestyle in those countries could be plausible explanations [10]. Another explanation could be the decreasing incidence of the left sides colonic tumors (sigmoid and descending colon) [10].

The current data showed that the mean age of the studied population was 51.1 years, with near half of the affected patients aged 50 years or less with no difference between males and females, our finding are going with researchers from Central Sudan [11] who concluded that $43.8 \%$ of $\mathrm{Ca}$ colon patients were below 50 years old, and male to female ratio of 1: 1.02

The current study reported that rectal bleeding and altered bowel habit were the commonest presentations among patients with colonic cancer in accordance with Mohamed et al. [12] who conducted a study in Sudan and concluded change in the bowel habit and rectal bleeding in $90.5 \%$, and $84 \%$ respectively.

Previous researchers [12, 13] observed that adenocarcinoma was the commonest histopathology among patients with $\mathrm{Ca}$ colon, in similarity with the present data in which $100 \%$ of patients were diagnosed with adenocarcinoma.

In the present study family history of colorectal cancer was evident in $16.7 \%$ (colonic cancer $10 \%$ and hereditary polyposis coli in $6.7 \%$ ) similar to Taha et al. [11] who conducted a study in Khartoum Sudan and reported a family history in $15.06 \%$ of cases.

The symptomatology overlap between the irritable bowel syndrome a common functional gastrointestinal disorder and subtle colonic cancer was previously documented [14], the over diagnosis of irritable bowel syndrome and the reliance on the history only for diagnosis could lead to the delayed presentation and hence poor prognosis of colorectal cancer. In the current study, chronic constipation, and increase frequency of stool (both are standard features of the irritable bowel syndrome) were reported in nearly two-thirds, and one-third of the study sample respectively, furthermore a considerable number of our patients presented with late features: Abdominal mass in 23.3\%, and intestinal obstruction in $20 \%$. Physicians may need to do the examination of the pack-passages and simple proctosigmoidoscopy for the early detection of the cancer of the commoner left colonic cancer.

\section{Conclusion}

The right shift of the colonic cancer was not observed in Sudan. The is a tendency towards younger age group; the commonest presentations were rectal bleeding and change in bowel habits. The Adenocarcinoma was the most frequent histopathological pattern.

\section{References}

[1] Amany Elamin, Muntaser E Ibrahim, Dafalla Abuidris, Kamal Eldin H Mohamed, and Sulma Ibrahim Mohammed. Part I: cancer in Sudan - burden, distribution, and trends breast, gynecological, and prostate cancers. Cancer Med. 2015 Mar; 4 (3): 447-456.

[2] Chalya PL, McHembe MD, Mabula JB, Rambau PF, Jaka H, Koy M, Mkongo E, Masalu N. Clinicopathological patterns and challenges of management of colorectal cancer in a resourcelimited setting: a Tanzanian experience. World J SurgOncol. 2013 Apr 18; 11: 88. doi: 10.1186/1477-7819-11-88.

[3] World Health Organization, author. Cancer. Fact Sheet (No. 297) Geneva:

2006.http://www.who.int/mediacentre/factsheets/fs297/en/prin t.html.

[4] Intisar E Saeed, Hsin-Yi Weng, Kamal H Mohamed, and SulmaMohammed. Cancer incidence in Khartoum, Sudan: first results from the Cancer Registry, 2009-2010. Cancer Med. 2014 Aug; 3 (4): 1075-1084.

[5] ParthPatela, Prithwish De. Trends in colorectal cancer incidence and related lifestyle risk factors in15-49-year-olds in Canada, 1969-2010. Cancer Epidemiology 42 (2016) 90-100.

[6] Khan T, Kayani N, Ahmad R, Usman A, Bhurgri A, Bashir I, Hasan SH, Zaidi S, Bhurgri Y. Incidence and current trends of colorectal malignancies in an unscreened, low risk Pakistan population. Asian Pac J Cancer Prev.2011; 12 (3): 703-8.

[7] Group WB. 2012. Washington, DC, USA World Bank Publications World Development Indicators 2012.

[8] Ghahremani GG, Dowlatshahi K. Colorectal carcinomas: diagnostic implications of their changing frequency and anatomic distribution. World J Surg. 1989 May-Jun; 13 (3): 321-4; discussion 324-5. 
[9] Ponz de Leon M, Marino M, Benatti P, Rossi G, Menigatti M, Pedroni M, Di Gregorio C, Losi L, Borghi F, Scarselli A, Ponti G, Roncari B, Zangardi G, Abbati G, Ascari E, Roncucci L. Trend of incidence, subsite distribution and staging of colorectal neoplasms in the 15-year experience of a specialised cancer registry. Ann Oncol. 2004 Jun; 15 (6): 940-6.

[10] Rabeneck L, Davila JA, El-Serag HB. Is there a true "shift" to the right colon in the incidence of colorectal cancer? Am J Gastroenterol. 2003 Jun; 98 (6): 1400-9.

[11] Taha MO, Abdalla AA, Mohamed RS. Pattern \& presentation of colorectal cancer in central Sudan, a retrospective descriptive study, 2010-2012. Afr Health Sci. 2015 Jun; 15 (2): $576-580$
[12] Mohammed M, Mussad A, Eltayeb E, A elaziz M. Clorectal carcinoma in Sudanese patients. International journal of medicine 2015; 3 (2): 98-102

[13] Albasri A, Yosef H, Hussainy AS, Sultan SA, Alhujaily A. Histopathological features of colorectal cancer in Al-Madinah region of Saudi Arabia: 8 years experience. Asian Pac J Cancer Prev. 2014; 15 (7): 3133-7.

[14] Nørgaard M, Farkas DK, Pedersen L, Erichsen R, de la Cour ZD, Gregersen H, Sørensen HT. Irritable bowel syndrome and risk of colorectal cancer: a Danish nationwide cohort study. $\mathrm{Br}$ J Cancer. 2011 Mar 29; 104 (7): 1202-6. doi: 10.1038/bjc.2011.65. Epub 2011 Feb 22. 